Article

\title{
An Extended EDAS Method with a Single-Valued Complex Neutrosophic Set and Its Application in Green Supplier Selection
}

\author{
Dongsheng Xu, Xiangxiang Cui * and Huaxiang Xian \\ School of Science, Southwest Petroleum University, Chengdu 610500, China; \\ xudongsheng1976@163.com (D.X.); xhx815242946@163.com (H.X.) \\ * Correspondence: 15608198802@163.com
}

Received: 31 December 2019; Accepted: 15 February 2020; Published: 19 February 2020

check for updates

\begin{abstract}
The single-valued complex neutrosophic set is a useful tool for handling the data with uncertainty and periodicity. In this paper, a single-valued complex neutrosophic EDAS (evaluation based on distance from average slution) model has been established and applied in green supplier selection. Firstly, the definition of single-valued complex neutrosophic set and corresponding operational laws are briefly introduced. Next, to fuse overall single-valued complex neutrosophic information, the SVCNEWA and SVCNEWG operators based on single-valued complex neutrosophic set, Einstein product and sum are proposed. Furthermore, the single-valued complex neutrosophic EDAS model has been established and all computing steps have been depicted in detail. Finally, a numerical example of green supplier selection and a comparison analysis have been given to illustrate the practicality and effectiveness of this new model.
\end{abstract}

Keywords: single-valued complex neutrosophic set; EDAS method; Einstein operator; multi-attribute group decision making problem

\section{Introduction}

With the growth of the world economy, more and more companies are being founded. Some of the most significant competition among modern enterprises is in their supply chains, and a key of supply chain management is supplier selection. Suppliers play an important role in high quality products and customer satisfaction. A preeminent supplier can improve the competitiveness of the enterprise [1]. Meanwhile, enterprises should consider significant environmental issues, such as the green effect, and stress suffered from the government, associations and the public [2]. So, green supplier selection (GSS) has been proposed, which is a construct that can supervise supplier performance along with green technical standards [3], and green supply chain management (GSCM) [4] as become an emerging field whose aim is to find a balance between the economy and environment. Numerous researches and scholars have studied this popular topic over the past several years. Zhang et al. [5] established a nonlinear multi-objective optimization model to deal with GSS problem and used a Pareto genetic algorithm to solve the problem. Hosseini and Barker [6] developed some resilience-based supplier selection criteria and a Bayesian network to present an innovative decision method for GSS which can address the risk and uncertainty in decision making problem.

In practical GSCM, supply chain managers need to consider all suppliers with many conflicting attributes, and consider the trade-off to select the optimal supplier(s). Therefore, GSS is commonly regarded as a multi-attribute group decision making (MAGDM) problem. Up to now, there are many researchers have studied the issue. Based on the best-worst method (BWM) and Vlsekriterijumska Optimizacija I Kompromisno Resenje (VIKOR) technique, Wu et al. [7] proposed a 
creative methodology to address GSS problem under interval type-2 fuzzy environment. Liu et al. [8] proposed an innovative GSS method by combining quality function deployment with the partitioned Bonferroni mean operator in interval type-2 fuzzy environment to solve a bike-share case. Qin et al. [9] proposed an extended TODIM multi-criteria group decision making method for green supplier selection under interval type-2 fuzzy environment.

For the MAGDM problem, there are many classical methods, such as TODIM [10], TOPSIS [11], VIKOR [12] and other methods [13-15]. Evaluation based on the distance from average solution (EDAS) method is an innovative decision making method, initially proposed by Ghorabaee et al. [16]. Compared with the compromise methods such as TOPSIS and VIKOR which need to calculate the distance from positive ideal solution (PIS) and negative ideal solution (NIS), EDAS owns its peculiar superiority of only considering distance from an average solution (AV) which can simplify the calculation procedure. The aim of EDAS method is to find the best one form a series of alternatives by using the positive distance from average (PDA) and the negative distance from average (NDA). In recent years, it has been studied by many researchers and scholars. Ghorabaee et al. [17] proposed an extended EDAS method to study the application of supplier selection. Kahraman et al. [18] proposed a new EDAS method with intuitionistic fuzzy number and applied it in the selection of solid waste disposal site. Karasan [19] extended EDAS method into an interval-valued neutrosophic set and applied it to the prioritization of United Nations national sustainable development goals.

However, due to the vagueness of human thinking and the complexity and uncertainty of objective things, it is difficult for decision makers to express an evaluated attribute with a crisp value. So neutrosophic set (NS) as an extension of fuzzy set (FS) [20] and intuitionistic fuzzy sets (IFS) [21] were originally proposed by Smarandache [22]. But, NS was mainly put forward from a philosophical viewpoint, which is difficult to be applied in the field of science and engineering. So single-valued neutrosophic set (SVNS) [23], interval neutrosophic set (INS) [24], simplified neutrosophic set (SNS) [25] and multi-valued neutrosophic set (MVNS) [26] were proposed by researchers. With the generation of "big data," which has the characteristics of uncertainty and periodicity, in order to handle this case, complex neutrosophic set (CNS) was proposed by Ali and Smarandache [27] who introduced the concept of CNS and discussed some properties of the CNS and set theoretic operations, and then applied them in signal processing. Later on, CNS became a new topic of neutrosophic theory. Al-Quran et al. [28] introduced the complex neutrosophic relation includes inverse, complement and composition with corresponding theorems and properties. Complex neutrosophic graphs of type 1 and some corresponding theoretical results were proposed by Quek et al. [29].

Information aggregation operators play a significant role in the MAGDM problem; they can fuse multiple values into a single comprehensive value. The most common types of operators include the arithmetic- and geometric-weighted operators [30,31], order-weighted operators [32,33], generalized operators [34,35], Bonferroni mean operators [36], Heronian mean operators [37], Einstein operators [38], etc. To use the advantages of Einstein operations and a generalized weighted average operator, a generalized simplified neutrosophic number Einstein weighed aggregation (GSNNEWA) operator was proposed in [39]. Peng et al. [40] defined the operations of multi-valued neutrosophic numbers (MVNNs) based on Einstein operations; furthermore, the multi-valued neutrosophic power weighted average (MVNPWA) operator and the multi-valued neutrosophic power weighted geometric (MVNPWG) operator based on the operation laws were proposed and applied to the MAGDM problem. Zhao and Wei [41] proposed the intuitionistic fuzzy Einstein hybrid averaging (IFEHA) operator and the intuitionistic fuzzy Einstein hybrid geometrical (IFEHG) operator, and then applied them in MADM.

The purpose of this paper is to establish an extended EDAS method model in single-valued complex neutrosophic environment and apply it to the selection of a green supplier. The specific arrangements of this article are structured as follows. In Section 2, we briefly introduce some concepts and definitions of SVCNS. In Section 3, we propose new operational rules of SVCNS based on the Einstein operator, and then the SVCNEWA and SVCNEWG operators are proposed. The single-valued 
complex neutrosophic EDAS model is established and the computing steps are listed in Section 4. In Section 5, an example is given to illustrate the application of proposed method. In Section 6, a conclusion of this paper is given.

\section{Preliminaries}

\subsection{Single-Valued Neutrosophic Set}

Definition 1. [22] Let X be a space of points (objects), with a generic element in X denoted by $x$. A neutrosophic set $A$ in $X$ is characterized by a truth-membership function $T_{A}(x)$, an indeterminacy-membership function $I_{A}(x)$ and a falsity-membership function $F_{A}(x)$, where $T_{A}(x), I_{A}(x)$ and $F_{A}(x)$ are real standard or nonstandard subsets of $] 0^{-}, 1^{+}\left[\right.$; that is, $\left.T_{A}(x): X \rightarrow\right] 0^{-}, 1^{+}\left[, I_{A}(x): X \rightarrow\right] 0^{-}, 1^{+}\left[\right.$and $F_{A}(x):$ $X \rightarrow] 0^{-}, 1^{+}[$.

There is no restriction on the sum of $T_{X}, I_{x}$ and $F x$, so $0^{-} \leq \sup T_{A}(x)+\sup I_{A}(x)+\sup F_{A}(x) \leq 3^{+}$.

Definition 2. [23] Let X be a space of points (objects), with a generic element in X denoted by $x$. A single-valued neutrosophic set $A$ in $X$ is characterized by a truth-membership function $T_{A}(x)$, an indeterminacy-membership function $I_{A}(x)$ and a falsity-membership function $F_{A}(x)$ with $T_{A}(x), I_{A}(x), F_{A}(x) \in[0,1]$ for all $x \in X$. Then, a single-valued neutrosophic set $A$ can be denoted as

$$
A=\left\{\left\langle x, T_{A}(x), I_{A}(x), F_{A}(x)\right\rangle: x \in X\right\}
$$

with the condition $0 \leq T_{A}(x)+I_{A}(x)+F_{A}(x) \leq 3$.

\subsection{Single-Valued Complex Neutrosophic Set}

Definition 3. [27] Let $X$ be a space of points (objects), with a generic element in $X$ denoted by $x$. A single-valued complex neutrosophic set $S$ in $X$ is characterized by a truth-membership function $T_{S}(x)$, an indeterminacy-membership function $I_{S}(x)$ and a falsity-membership function $F_{S}(x)$ that assigns a complex-valued membership grade to $T_{S}(x), I_{S}(x)$ and $F_{S}(x)$ for all $x \in X$. The values of $T_{S}(x), I_{S}(x)$, $F_{S}(x)$ and their sum may fall within the unit circle in the complex plane; the former is of the following form:

$$
T_{S}(x)=p_{S}(x) \cdot e^{j \omega_{S}(x)}, I_{S}(x)=q_{S}(x) \cdot e^{j \psi_{S}(x)}, F_{S}(x)=r_{S}(x) \cdot e^{j \phi_{S}(x)}
$$

where $\sqrt{j}=-1, p_{S}(x), q_{S}(x), r_{S}(x)$ and $\omega_{S}(x), \psi_{S}(x), \phi_{S}(x)$ are real values and $p_{S}(x), q_{S}(x), r_{S}(x) \in$ $[0,1]$ such that $0 \leq p_{S}(x)+q_{S}(x)+r_{S}(x) \leq 3$. For convenience a single-valued complex neutrosophic set $S$ can be represented in set form as:

$$
S=\left\{\left\langle x, T_{S}(x), I_{S}(x), F_{S}(x)\right\rangle: x \in X\right\}
$$

and a single-valued neutrosophic number (SVCNN) can be denoted as $S=\left\langle T_{S}, I_{S}, F_{S}\right\rangle$ which is a basic unit of single-valued complex neutrosophic set.

Definition 4. [28] Let $S=\left\{\left\langle x, T_{S}(x), I_{S}(x), F_{S}(x)\right\rangle: x \in X\right\}$ be a SVCNS in X. Then, the complement of $S$ is denoted as $c(S)$ and specified by functions:

$$
\begin{gathered}
T_{c(S)}(x)=p_{c(S)} \cdot e^{j \omega_{c(S)}(x)}=r_{S}(x) \cdot e^{j\left(2 \pi-\omega_{S}(x)\right)} \\
I_{c(S)}(x)=q_{c(S)} \cdot e^{j \psi_{c(S)}(x)}=\left(1-q_{S}(x)\right) \cdot e^{j\left(2 \pi-\psi_{S}(x)\right)} \\
F_{c(S)}(x)=r_{c(S)} \cdot e^{j \phi_{c(S)}(x)}=p_{S}(x) \cdot e^{j\left(2 \pi-\phi_{S}(x)\right)}
\end{gathered}
$$


Definition 5. Let $A$ and $B$ be two SVCNSs which are defined by $T_{A}(x)=p_{A}(x) \cdot e^{j \omega_{A}(x)}, I_{A}(x)=$ $q_{A}(x) \cdot e^{j \psi_{A}(x)}, F_{A}(x)=r_{A}(x) \cdot e^{j \phi_{A}(x)}$ and $T_{B}(x)=p_{B}(x) \cdot e^{j \omega_{B}(x)}, I_{B}(x)=q_{B}(x) \cdot e^{j \psi_{B}(x)}, F_{B}(x)=$ $r_{B}(x) \cdot e^{j \phi_{B}(x)}$, respectively. Then, the operational rules of SVCNSs are defined as follows:

(1) The sum of $A$ and $B$, denoted as $A \oplus B$, is defined as

$$
\begin{gathered}
T_{A \oplus B}(x)=\left(p_{A}(x)+p_{B}(x)-p_{A}(x) p_{B}(x)\right) \cdot e^{j 2 \pi\left(\frac{\omega_{A}(x)}{2 \pi}+\frac{\omega_{B}(x)}{2 \pi}-\frac{\omega_{A}(x) \omega_{B}(x)}{(2 \pi)^{2}}\right)} \\
I_{A \oplus B}(x)=\left(q_{A}(x) q_{B}(x)\right) \cdot e^{j 2 \pi\left(\frac{\psi_{A}(x)}{2 \pi} \cdot \frac{\psi_{B}(x)}{2 \pi}\right)} \\
F_{A \oplus B}(x)=\left(r_{A}(x) r_{B}(x)\right) \cdot e^{j 2 \pi\left(\frac{\phi_{A}(x)}{2 \pi} \cdot \frac{\phi_{B}(x)}{2 \pi}\right)}
\end{gathered}
$$

(2) The product of $A$ and $B$, denoted as $A \otimes B$, is defined as

$$
\begin{gathered}
T_{A \otimes B}(x)=\left(p_{A}(x) p_{B}(x)\right) \cdot e^{j 2 \pi\left(\frac{\omega_{A}(x)}{2 \pi} \cdot \frac{\omega_{B}(x)}{2 \pi}\right)} \\
I_{A \otimes B}(x)=\left(q_{A}(x)+q_{B}(x)-q_{A}(x) q_{B}(x)\right) \cdot e^{j 2 \pi\left(\frac{\psi_{A}(x)}{2 \pi}+\frac{\psi_{B}(x)}{2 \pi}-\frac{\psi_{A}(x) \psi_{B}(x)}{(2 \pi)^{2}}\right)} \\
F_{A \otimes B}(x)=\left(r_{A}(x)+r_{B}(x)-r_{A}(x) r_{B}(x)\right) \cdot e^{j 2 \pi\left(\frac{\phi_{A}(x)}{2 \pi}+\frac{\phi_{B}(x)}{2 \pi}-\frac{\phi_{A}(x) \phi_{B}(x)}{(2 \pi)^{2}}\right)}
\end{gathered}
$$

(3) The scalar multiplication of $A$ is a single-valued complex neutrosophic set denoted as $C=\lambda A(\lambda>0)$, defined as:

$$
\begin{gathered}
T_{C}(x)=\left(1-\left(1-p_{A}(x)\right)^{\lambda}\right) \cdot e^{j 2 \pi\left(1-\left(1-\frac{\omega_{A}(x)}{2 \pi}\right)^{\lambda}\right)} \\
I_{C}(x)=\left(\left(q_{A}(x)\right)^{\lambda}\right) \cdot e^{j 2 \pi\left(\frac{\psi_{A}(x)}{2 \pi}\right)^{\lambda}} \\
F_{C}(x)=\left(\left(r_{A}(x)\right)^{\lambda}\right) \cdot e^{j 2 \pi\left(\frac{\phi_{A}(x)}{2 \pi}\right)^{\lambda}}
\end{gathered}
$$

(4) The power of $A$ is denoted as $D=(A)^{\lambda}(\lambda>0)$, and defined as:

$$
\begin{gathered}
T_{D}(x)=\left(\left(p_{A}(x)\right)^{\lambda}\right) \cdot e^{j 2 \pi\left(\frac{\omega_{A}(x)}{2 \pi}\right)^{\lambda}} \\
I_{D}(x)=\left(1-\left(1-q_{A}(x)\right)^{\lambda}\right) \cdot e^{j 2 \pi\left(1-\left(1-\frac{\psi_{A}(x)}{2 \pi}\right)^{\lambda}\right)} \\
F_{D}(x)=\left(1-\left(1-r_{A}(x)\right)^{\lambda}\right) \cdot e^{j 2 \pi\left(1-\left(1-\frac{\phi_{A}(x)}{2 \pi}\right)^{\lambda}\right)}
\end{gathered}
$$

Definition 6. Let $X=\left\{x_{1}, x_{2}, \cdots, x_{n}\right\}$ be a universal of objects, $A$ and $B$ be two SVCNSs in $X$, and then the normalized Hamming distance between $A$ and $B$ is:

$$
\begin{aligned}
D(A, B)= & \frac{1}{6 n} \sum_{j=1}^{n}\left\{\left|T_{A}\left(x_{j}\right)-T_{B}\left(x_{j}\right)\right|+\left|I_{A}\left(x_{j}\right)-I_{B}\left(x_{j}\right)\right|+\left|F_{A}\left(x_{j}\right)-F_{B}\left(x_{j}\right)\right|+\right. \\
& \left.\frac{1}{2 \pi}\left(\left|\omega_{A}\left(x_{j}\right)-\omega_{B}\left(x_{j}\right)\right|+\left|\psi_{A}\left(x_{j}\right)-\psi_{B}\left(x_{j}\right)\right|+\left|\phi_{A}\left(x_{j}\right)-\phi_{B}\left(x_{j}\right)\right|\right)\right\}
\end{aligned}
$$

Definition 7. Let $A$ be a SVCNN; then, the score function $S(A)$ of $A$ is defined as:

$$
S(A)=\frac{1}{6}\left(\left(2+T_{A}-I_{A}-F_{A}\right)+\frac{1}{2 \pi}\left(4 \pi+\omega_{A}-\psi_{A}-\phi_{A}\right)\right)
$$


Definition 8. Let $A$ be a SVCNN, and then the score function $H(A)$ of $A$ is defined as:

$$
H(A)=\frac{1}{2}\left(\left(T_{A}-F_{A}\right)+\frac{1}{2 \pi}\left(\omega_{A}-\phi_{A}\right)\right)
$$

Definition 9. let $A_{1}$ and $A_{2}$ be two SVCNNs, and $S$ be the score function, $H$ be the accuracy function. If $S\left(A_{1}\right)<S\left(A_{2}\right)$, then $A_{1}<A_{2} ;$ if $S\left(A_{1}\right)=S\left(A_{2}\right)$, then

(1) If $H\left(A_{1}\right)<H\left(A_{2}\right)$, then $A_{1}<A_{2}$;

(2) If $H\left(A_{1}\right)=H\left(A_{2}\right)$, then $A_{1}=A_{2}$.

\section{The Einstein Operator with SVCNNs}

The Einstein operator plays a significant role in as an aggregation operator. It consists of the Einstein product $\otimes_{E}$ and the Einstein sum $\oplus_{E}$, where $\otimes_{E}$ is a t-norm and $\oplus_{E}$ is a t-conorm. They are defined as follows: [38]

$$
\begin{gathered}
\Gamma_{E}(x, y)=x \otimes_{E} y=\frac{x y}{1+(1-x)(1-y)} \\
\Gamma_{E}^{*}(x, y)=x \oplus_{E} y=\frac{x+y}{1+x y}
\end{gathered}
$$

In the following, we shall propose single-valued complex neutrosophic set operational rules based on the Einstein operator.

Definition 10. Let $A$ and $B$ be two SVCNSs which are defined by $T_{A}(x)=p_{A}(x) \cdot e^{j \omega_{A}(x)}, I_{A}(x)=$ $q_{A}(x) \cdot e^{j \psi_{A}(x)}, F_{A}(x)=r_{A}(x) \cdot e^{j \phi_{A}(x)}$ and $T_{B}(x)=p_{B}(x) \cdot e^{j \omega_{B}(x)}, I_{B}(x)=q_{B}(x) \cdot e^{j \psi_{B}(x)}, F_{B}(x)=$ $r_{B}(x) \cdot e^{j \phi_{B}(x)}$, respectively. Then, the Einstein operational rules of SVCNSs are defined as follows:

(1)

$$
\begin{aligned}
A \oplus_{E} B= & \left\langle\frac{p_{A}+p_{B}}{1+p_{A} p_{B}} \cdot e^{j 2 \pi\left(\frac{\frac{\omega_{A}}{2 \pi}+\frac{\omega_{B}}{2 \pi}}{1+\frac{\omega_{A} \omega_{B}}{(2 \pi)^{2}}}\right)}, \frac{q_{A} q_{B}}{1+\left(1-q_{A}\right)\left(1-q_{B}\right)} \cdot e^{j 2 \pi\left(\frac{\frac{\psi_{A} \psi_{B}}{(2 \pi)^{2}}}{1+\left(1-\frac{\psi_{A}}{2 \pi}\right)\left(1-\frac{\psi_{B}}{2 \pi}\right)}\right),}\right. \\
& \left.\frac{r_{A} r_{B}}{1+\left(1-r_{A}\right)\left(1-r_{B}\right)} \cdot e^{j 2 \pi\left(\frac{\frac{\phi_{A} \phi_{B}}{(2 \pi)^{2}}}{1+\left(1-\frac{\phi_{A}}{2 \pi}\right)\left(1-\frac{\phi_{B}}{2 \pi}\right)}\right)}\right\rangle
\end{aligned}
$$

(2)

$$
\begin{aligned}
A \otimes_{E} B= & \left\langle\frac{p_{A} p_{B}}{1+\left(1-p_{A}\right)\left(1-p_{B}\right)} \cdot e^{j 2 \pi\left(\frac{\frac{\omega_{A} \omega_{B}}{(2 \pi)^{2}}}{1+\left(1-\frac{\omega_{A}}{2 \pi}\right)\left(1-\frac{\omega_{B}}{2 \pi}\right)}\right)}, \frac{q_{A}+q_{B}}{1+q_{A} q_{B}} \cdot e^{j 2 \pi\left(\frac{\frac{\psi_{A}}{2 \pi}+\frac{\psi_{B}}{2 \pi}}{1+\frac{\psi_{A} \psi_{B}}{(2 \pi)^{2}}}\right),}\right. \\
& \left.\frac{r_{A}+r_{B}}{1+r_{A} r_{B}} \cdot e^{j 2 \pi\left(\frac{\frac{\phi_{A}}{2 \pi}+\frac{\phi_{B}}{2 \pi}}{1+\frac{\phi_{A} \phi_{B}}{(2 \pi)^{2}}}\right)}\right\rangle
\end{aligned}
$$


(3)

$$
\begin{aligned}
\lambda A= & \left\langle\frac{\left(1+p_{A}\right)^{\lambda}-\left(1-p_{A}\right)^{\lambda}}{\left(1+p_{A}\right)^{\lambda}+\left(1-p_{A}\right)^{\lambda}} \cdot e^{j 2 \pi\left(\frac{\left(1+\frac{\omega_{A}}{2 \pi}\right)^{\lambda}-\left(1-\frac{\omega_{A}}{2 \pi}\right)^{\lambda}}{\left(1+\frac{\omega_{A}}{2 \pi}\right)^{\lambda}+\left(1-\frac{\omega_{A}}{2 \pi}\right)^{\lambda}}\right)},\right. \\
& \frac{2\left(q_{A}\right)^{\lambda}}{\left(2-q_{A}\right)^{\lambda}+\left(q_{A}\right)^{\lambda}} \cdot e^{j 2 \pi\left(\frac{2\left(\frac{\psi_{A}}{2 \pi}\right)^{\lambda}}{\left(2-\frac{\psi_{A}}{2 \pi}\right)^{\lambda}+\left(\frac{\psi_{A}}{2 \pi}\right)^{\lambda}}\right)}, \frac{2\left(r_{A}\right)^{\lambda}}{\left(2-r_{A}\right)^{\lambda}+\left(r_{A}\right)^{\lambda}} \cdot e^{\left.j 2 \pi\left(\frac{2\left(\frac{\phi_{A}}{2 \pi}\right)^{\lambda}}{\left(2-\frac{\phi_{A}}{2 \pi}\right)^{\lambda}+\left(\frac{\phi_{A}}{2 \pi}\right)^{\lambda}}\right)\right\rangle}
\end{aligned}
$$

(4)

$$
\begin{aligned}
A^{\lambda}= & \left\langle\frac{2\left(p_{A}\right)^{\lambda}}{\left(2-p_{A}\right)^{\lambda}+\left(p_{A}\right)^{\lambda}} \cdot e^{j 2 \pi\left(\frac{2\left(\frac{\omega_{A}}{2 \pi}\right)^{\lambda}}{\left(2-\frac{\omega_{A}}{2 \pi}\right)^{\lambda}+\left(\frac{\omega_{A}}{2 \pi}\right)^{\lambda}}\right),}\right. \\
& \frac{\left(1+q_{A}\right)^{\lambda}-\left(1-q_{A}\right)^{\lambda}}{\left(1+q_{A}\right)^{\lambda}+\left(1-q_{A}\right)^{\lambda}} \cdot e^{j 2 \pi\left(\frac{\left(1+\frac{\psi_{A}}{2 \pi}\right)^{\lambda}-\left(1-\frac{\psi_{A}}{2 \pi}\right)^{\lambda}}{\left(1+\frac{\psi_{A}}{2 \pi}\right)^{\lambda}+\left(1-\frac{\psi_{A}}{2 \pi}\right)^{\lambda}}\right),} \\
& \left.\frac{\left(1+r_{A}\right)^{\lambda}-\left(1-r_{A}\right)^{\lambda}}{\left(1+r_{A}\right)^{\lambda}+\left(1-r_{A}\right)^{\lambda}} \cdot e^{j 2 \pi\left(\frac{\left(1+\frac{\phi_{A}}{2 \pi}\right)^{\lambda}-\left(1-\frac{\phi_{A}}{2 \pi}\right)^{\lambda}}{\left(1+\frac{\phi_{A}}{2 \pi}\right)^{\lambda}+\left(1-\frac{\phi_{A}}{2 \pi}\right)^{\lambda}}\right)}\right\rangle
\end{aligned}
$$

Definition 11. Let $A_{i}=\left\langle T_{i} \cdot e^{j \omega_{i}}, I_{i} \cdot e^{j \psi_{i}}, F_{i} \cdot e^{j \phi_{i}}\right\rangle(i=1,2, \cdots, n)$ be a collection of SVCNNs, the single-valued, complex, neutrosophic, Einstein-weighted average (SVCNEWA) operator and single-valued, complex, neutrosophic, Einstein-weighted geometric (SVCNEWG) operator can be defined as follows:

$$
\operatorname{SVCNEWA}\left(A_{1}, A_{2}, \cdots, A_{n}\right)=w_{1} A_{1} \oplus_{E} w_{2} A_{2} \oplus_{E} \cdots \oplus_{E} w_{n} A_{n}=\oplus_{i=1}^{n}\left(w_{i} A_{i}\right)
$$

and

$$
\operatorname{SVCNEWG}\left(A_{1}, A_{2}, \cdots, A_{n}\right)=w_{1} A_{1} \otimes_{E} w_{2} A_{2} \otimes_{E} \cdots \otimes_{E} w_{n} A_{n}=\otimes_{i=1}^{n}\left(A_{i}\right)^{w_{i}}
$$

where $w_{i}$ is the weight of $A_{i}$ with the condition $w_{i} \in[0,1]$ and $\sum_{i=1}^{n} w_{i}=1$.

Theorem 1. Let $A_{i}=\left\langle T_{i} \cdot e^{j \omega_{i}}, I_{i} \cdot e^{j \psi_{i}}, F_{i} \cdot e^{j \phi_{i}}\right\rangle(i=1,2, \cdots, n)$ be a collection of SVCNNs, then the operation results by SVCNEWA and SVCNEWG operators are also a SVCNN where:

$$
\begin{aligned}
& \operatorname{SVCNEWA}\left(A_{1}, A_{2}, \cdots, A_{n}\right)=\underset{i=1}{\oplus_{E}}\left(w_{i} A_{i}\right) \\
& =\left(\frac{\prod_{i=1}^{n}\left(1+T_{i}\right)^{w_{i}}-\prod_{i=1}^{n}\left(1-T_{i}\right)^{w_{i}}}{\prod_{i=1}^{n}\left(1+T_{i}\right)^{w_{i}}+\prod_{i=1}^{n}\left(1-T_{i}\right)^{w_{i}}} \cdot e^{j 2 \pi\left(\frac{\prod_{i=1}^{n}\left(1+\frac{\omega_{i}}{2 \pi}\right)^{w_{i}}-\prod_{i=1}^{n}\left(1-\frac{\omega_{i}}{2 \pi}\right)^{w_{i}}}{\prod_{i=1}^{n}\left(1+\frac{w_{i}}{2 \pi}\right)^{w_{i}}+\prod_{i=1}^{w_{i}}\left(1-\frac{\omega_{i}}{2 \pi}\right)^{w_{i}}}\right)}, \frac{2 \prod_{i=1}^{n}\left(I_{i}\right)^{w_{i}}}{\prod_{i=1}^{n}\left(2-I_{i}\right)^{w_{i}}+\prod_{i=1}^{n}\left(I_{i}\right)^{w_{i}}} \cdot e^{j 2 \pi\left(\frac{\prod_{i=1}^{n}\left(\frac{\psi_{i}}{2 \pi}\right)^{w_{i}}}{\prod_{i=1}^{n}\left(2-\frac{\psi_{i}}{2 \pi}\right)^{w_{i}}+\prod_{i=1}^{n}\left(\frac{\psi_{i}}{2 \pi}\right)^{w_{i}}}\right)},\right. \\
& \left.\frac{2 \prod_{i=1}^{n}\left(F_{i}\right)^{w_{i}}}{\prod_{i=1}^{n}\left(2-F_{i}\right)^{w_{i}}+\prod_{i=1}^{n}\left(F_{i}\right)^{w_{i}}} \cdot e^{j 2 \pi\left(\frac{2 \prod_{i=1}^{n}\left(\frac{\phi_{i}}{2 \pi}\right)^{w_{i}}}{\prod_{i=1}^{n}\left(2-\frac{\phi_{i}}{2 \pi}\right)^{w_{i}}+\prod_{i=1}^{n}\left(\frac{\phi_{i}}{2 \pi}\right)^{w_{i}}}\right)}\right)
\end{aligned}
$$


and

$$
\begin{aligned}
& \operatorname{SVCNEWG}\left(A_{1}, A_{2}, \cdots, A_{n}\right)=\otimes_{i=1}^{n}\left(A_{i}\right)^{w_{i}}
\end{aligned}
$$

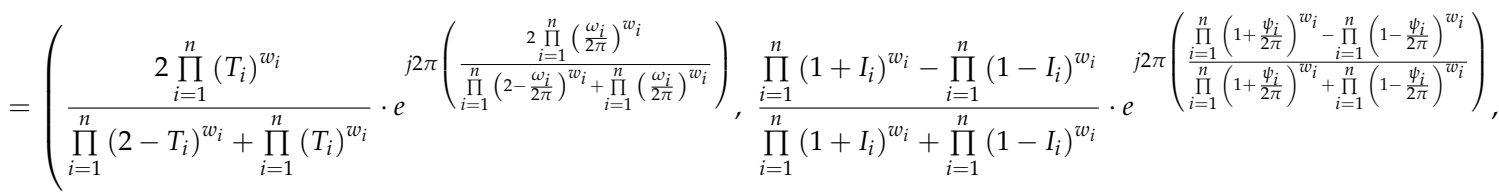

$$
\begin{aligned}
& \left.\frac{\prod_{i=1}^{n}\left(1+F_{i}\right)^{w_{i}}-\prod_{i=1}^{n}\left(1-F_{i}\right)^{w_{i}}}{\prod_{i=1}^{n}\left(1+F_{i}\right)^{w_{i}}+\prod_{i=1}^{n}\left(1-F_{i}\right)^{w_{i}}} \cdot e^{j 2 \pi\left(\frac{\prod_{i=1}^{n}\left(1+\frac{\phi_{i}}{2 \pi}\right)^{w_{i}}-\prod_{i=1}^{n}\left(1-\frac{\phi_{i}}{2 \pi}\right)^{w_{i}}}{\prod_{i=1}^{n}\left(1+\frac{\phi_{i}}{2 \pi}\right)^{w_{i}}+\prod_{i=1}^{n}\left(1-\frac{\phi_{i}}{2 \pi}\right)^{w_{i}}}\right)}\right)
\end{aligned}
$$

Proof. In this part, we can prove the above theorem by mathematical induction.

(1) When $n=2$, we have

$$
\begin{aligned}
& \operatorname{SVCNEWA}\left(A_{1}, A_{2}\right)=w_{1} A_{1} \oplus_{E} w_{2} A_{2} \\
& =\left\langle\frac{\left(1+T_{1}\right)^{w_{1}}-\left(1-T_{1}\right)^{2 w_{1}}}{\left(1+T_{1}\right)^{w_{1}}+\left(1-T_{1}\right)^{w_{1}}} \cdot e^{j 2 \pi\left(\frac{\left(1+\frac{\omega_{1}}{2 \pi}\right)^{w_{1}}-\left(1-\frac{\omega_{1}}{2 \pi}\right)^{w_{1}}}{\left(1+\frac{\omega_{1}}{2 \pi}\right)^{w_{1}}+\left(1-\frac{-\omega_{1}}{2 \pi}\right)^{w_{1}}}\right)}, \frac{2\left(I_{1}\right)^{w_{1}}}{\left(2-I_{1}\right)^{w_{1}}+\left(I_{1}\right)^{w_{1}}} \cdot e^{j 2 \pi\left(\frac{2\left(\frac{\psi_{1}}{2 \pi}\right)^{w_{1}}}{\left(2-\frac{\psi_{1}}{2 \pi}\right)^{w_{1}}+\left(\frac{\psi_{1}}{2 \pi}\right)^{w_{1}}}\right)},\right. \\
& \left.\frac{2\left(F_{1}\right)^{w_{1}}}{\left(2-F_{1}\right)^{w_{1}}+\left(F_{1}\right)^{w_{1}}} \cdot e^{j 2 \pi\left(\frac{2\left(\frac{\phi_{1}}{2 \pi}\right)^{w_{1}}}{\left(2-\frac{\phi_{1}}{2 \pi}\right)^{w_{1}}+\left(\frac{\phi_{1}}{2 \pi}\right)^{w_{1}}}\right)}\right\rangle \oplus_{E}
\end{aligned}
$$

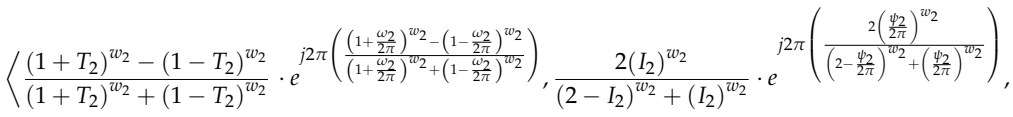

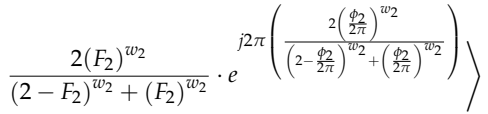

$$
\begin{aligned}
& =\left(\frac{\prod_{i=1}^{2}\left(1+T_{i}\right)^{w_{i}}-\prod_{i=1}^{2}\left(1-T_{i}\right)^{w_{i}}}{\prod_{i=1}^{2}\left(1+T_{i}\right)^{w_{i}}+\prod_{i=1}^{2}\left(1-T_{i}\right)^{w_{i}}} \cdot e^{j 2 \pi\left(\frac{\prod_{i=1}^{2}\left(1+\frac{\omega_{i}}{2 \pi}\right)^{w_{i}}-\prod_{i=1}^{2}\left(1-\frac{\omega_{i}}{2 \pi}\right)^{w_{i}}}{\prod_{i=1}^{2}\left(1+\frac{\omega_{i}}{2 \pi}\right)^{w_{i}}+\prod_{i=1}^{2}\left(1-\frac{\omega_{i}}{2 \pi}\right)^{w_{i}}}\right)}, \frac{2 \prod_{i=1}^{2}\left(I_{i}\right)^{w_{i}}}{\prod_{i=1}^{2}\left(2-I_{i}\right)^{w_{i}}+\prod_{i=1}^{2}\left(I_{i}\right)^{w_{i}}} \cdot e^{j 2 \pi\left(\frac{2 \prod_{i=1}^{2}\left(\frac{\Psi_{i}}{2 \pi}\right)^{w_{i}}}{\prod_{i=1}^{2}\left(2-\frac{\psi_{i}}{2 \pi}\right)^{w_{i}}+\prod_{i=1}^{2}\left(\frac{\varphi_{i}}{2 \pi}\right)^{w_{i}}}\right)},\right. \\
& \left.\frac{2 \prod_{i=1}^{2}\left(F_{i}\right)^{w_{i}}}{\prod_{i=1}^{2}\left(2-F_{i}\right)^{w_{i}}+\prod_{i=1}^{2}\left(F_{i}\right)^{w_{i}}} \cdot e^{j 2 \pi\left(\frac{2 \prod_{i=1}^{2}\left(\frac{\phi_{i}}{2 \pi}\right)^{w_{i}}}{\prod_{i=1}^{2}\left(2-\frac{\phi_{i}}{2 \pi}\right)^{w_{i}}+\prod_{i=1}^{2}\left(\frac{\phi_{i}}{2 \pi}\right)^{w_{i}}}\right)}\right)
\end{aligned}
$$

Therefore, when $n=2$, the equation is true.

(2) Assume that when $n=k$, Equation (10) is true. Then when $n=k+1$, we have

$$
\begin{aligned}
& \operatorname{SVCNEWA}\left(A_{1}, A_{2}, \cdots, A_{k}, A_{k+1}\right)=\operatorname{SVCNEWA}\left(A_{1}, A_{2}, \cdots, A_{k}\right) \oplus_{E} w_{k+1} A_{k+1} \\
& =\left(\frac{\prod_{i=1}^{n}\left(1+T_{i}\right)^{w_{i}}-\prod_{i=1}^{n}\left(1-T_{i}\right)^{w_{i}}}{\prod_{i=1}^{n}\left(1+T_{i}\right)^{w_{i}}+\prod_{i=1}^{n}\left(1-T_{i}\right)^{w_{i}}} \cdot e^{j 2 \pi\left(\frac{\prod_{i=1}^{n}\left(1+\frac{\omega_{i}}{2 \pi}\right)^{w_{i}}-\prod_{i=1}^{n}\left(1-\frac{\omega_{i}}{2 \pi}\right)^{w_{i}}}{\prod_{i=1}^{n}\left(1+\frac{\omega_{i}}{2 \pi}\right)^{w_{i}}+\prod_{i=1}^{n}\left(1-\frac{\omega_{i}}{2 \pi}\right)^{w_{i}}}\right)}, \frac{2 \prod_{i=1}^{n}\left(I_{i}\right)^{w_{i}}}{\prod_{i=1}^{n}\left(2-I_{i}\right)^{w_{i}}+\prod_{i=1}^{n}\left(I_{i}\right)^{w_{i}}} \cdot e^{j 2 \pi\left(\frac{2 \prod_{i=1}^{n}\left(\frac{\psi_{i}}{2 \pi}\right)^{w_{i}}}{\prod_{i=1}^{n}\left(2-\frac{\varphi_{i}}{2 \pi}\right)^{w_{i}}+\prod_{i=1}^{n}\left(\frac{\varphi_{i}}{2 \pi}\right)^{w_{i}}}\right)},\right. \\
& \left.\frac{2 \prod_{i=1}^{n}\left(F_{i}\right)^{w_{i}}}{\prod_{i=1}^{n}\left(2-F_{i}\right)^{w_{i}}+\prod_{i=1}^{n}\left(F_{i}\right)^{w_{i}}} \cdot e^{j 2 \pi\left(\frac{2 \prod_{i=1}^{n}\left(\frac{\phi_{i}}{w_{i} \pi}\right)^{w_{i}}}{\prod_{i=1}^{n}\left(2-\frac{\phi_{i}}{2 \pi}\right)^{w_{i}}+\prod_{i=1}^{n}\left(\frac{\phi_{i}}{2 \pi}\right)^{w_{i}}}\right)}\right) \oplus_{E}
\end{aligned}
$$

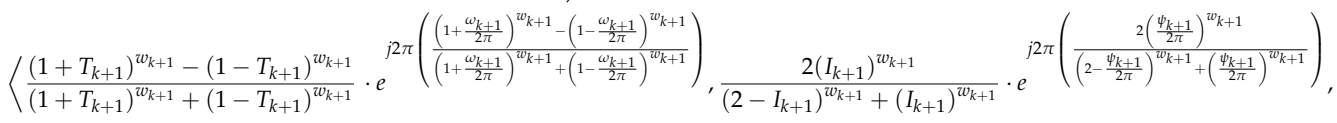




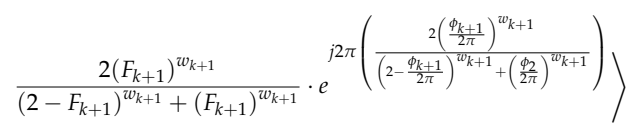

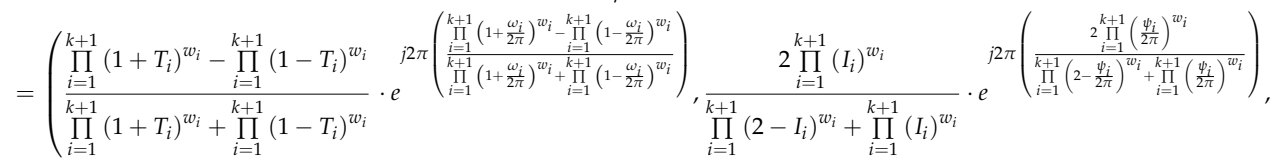

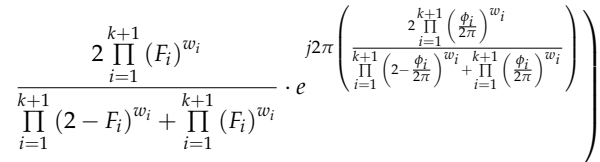

Thus, when $n=k+1$, the equation is true.

So we can calculate $\operatorname{SVCNEWA}\left(A_{1}, A_{2}, \cdots, A_{n}\right)=\oplus_{i=1}^{n}\left(w_{i} A_{i}\right)$ for any $n$. In the same way, we can obtain the form of SVCNEWG operator shown in Theorem 1.

\section{The EDAS Method with SVCNNs}

In this section, a MAGDM approach by combining the proposed operators and EDAS method is presented.

Suppose there is a committee of $r$ experts $\left\{E_{1}, E_{2}, \cdots, E_{r}\right\}$ with the weight vector of experts $v=\left\{v_{1}, v_{2}, \cdots, v_{r}\right\}$ which satisfies $v_{i} \in[0,1]$ and $\sum_{i=1}^{r} v_{i}=1$ is responsible for evaluating $m$ alternatives $\left\{A_{1}, A_{2}, \cdots, A_{m}\right\}$ under $n$ attributes $\left\{C_{1}, C_{2}, \cdots, C_{n}\right\}$ where the weight vectors of attribute are $w=$ $\left\{w_{1}, w_{2}, \cdots, w_{n}\right\}, w_{i} \in[0,1]$ and $\sum_{i=1}^{n} w_{i}=1$.

Step 1: Construct the evaluation matrix of expert $E_{d}$, and denote it as $A_{d}=\left[a_{i j}^{d}\right]_{m \times n}, i=$ $1,2, \cdots, m, j=1,2, \cdots, n, d=1,2, \cdots, r$ where $a_{i j}^{d}=\left\langle T_{i j}^{d} \cdot e^{j \omega_{i j}^{d}}, I_{i j}^{d} \cdot e^{j \psi_{i j}^{d}}, F_{i j}^{d} \cdot e^{j \phi_{i j}^{d}}\right\rangle$ is a SVCNN and represents the single-valued complex neutrosophic information of alternative $A_{i}$ versus attribute $C_{j}$ by expert $E_{d}$.

Step 2: Normalize the evaluation matrix $A_{d}=\left[a_{i j}^{d}\right]_{m \times n}$ into $A^{\prime}{ }_{d}=\left[a_{i j}^{\prime d}\right]_{m \times n}$

For benefit-type attributes $C_{j}, a_{i j}^{\prime d}=a_{i j}^{d}=\left\langle T_{i j}^{d} \cdot e^{j \omega_{i j}^{d}}, I_{i j}^{d} \cdot e^{j \psi_{i j}^{d}}, F_{i j}^{d} \cdot e^{j \phi_{i j}^{d}}\right\rangle$

For cost-type attributes $C_{j}, a_{i j}^{\prime d}=c\left(a_{i j}^{d}\right)=\left\langle F_{i j}^{d} \cdot e^{j\left(2 \pi-\omega_{i j}^{d}\right)},\left(1-I_{i j}^{d}\right) \cdot e^{j\left(2 \pi-\psi_{i j}^{d}\right)}, T_{i j}^{d} \cdot e^{j\left(2 \pi-\phi_{i j}^{d}\right)}\right\rangle$

Step 3: According to the normalized decision making matrix $A_{d}^{\prime}=\left[a_{i j}^{\prime d}\right]_{m \times n}$ and the weight vector $v=\left\{v_{1} \cdot v_{2}, \cdots, v_{r}\right\}$ of experts, we can fuse overall $a_{i j}^{\prime d}$ into $a_{i j}$ by using the SVCNEWA or SVCNEWG operator; then the aggregated decision making matrix $A=\left[a_{i j}\right]_{m \times n}$ with aggregated information is obtained and denoted as $A=\left[a_{i j}\right]_{m \times n}=\left[\left\langle T_{i j} \cdot e^{j \omega_{i j}}, I_{i j} \cdot e^{j \psi_{i j}}, F_{i j} \cdot e^{j \phi_{i j}}\right\rangle\right]_{m \times n}$.

Step 4: Compute the value of the average solution $A V=\left(A V_{j}\right)_{1 \times n}$ where $A V_{j}=\frac{1}{m} \underset{i=1}{\oplus} a_{i j}$.

Based on Definition 5, we can get

$$
\begin{aligned}
{\underset{i=1}{m}}_{i=1}^{m} a_{i j}= & \left\langle\left(1-\prod_{i=1}^{m}\left(1-T_{i j}\right)\right) \cdot e^{j 2 \pi\left(1-\prod_{i=1}^{m}\left(1-\frac{\omega_{i j}}{2 \pi}\right)\right)},\left(\prod_{i=1}^{m}\left(T_{i j}\right)\right) \cdot e^{j 2 \pi\left(\prod_{i=1}^{m}\left(\frac{\psi_{i j}}{2 \pi}\right)\right),}\right. \\
& \left.\left(\prod_{i=1}^{m}\left(F_{i j}\right)\right) \cdot e^{j 2 \pi\left(\prod_{i=1}^{m}\left(\frac{\phi_{i j}}{2 \pi}\right)\right)}\right\rangle
\end{aligned}
$$


So

$$
\begin{aligned}
A V_{j}= & \left\langle\left(1-\prod_{i=1}^{m}\left(1-T_{i j}\right)^{\frac{1}{m}}\right) \cdot e^{j 2 \pi\left(1-\prod_{i=1}^{m}\left(1-\frac{\omega_{i j}}{2 \pi}\right)^{\frac{1}{m}}\right)},\left(\prod_{i=1}^{m}\left(T_{i j}\right)^{\frac{1}{m}}\right) \cdot e^{j 2 \pi\left(\prod_{i=1}^{m}\left(\frac{\psi_{i j}}{2 \pi}\right)^{\frac{1}{m}}\right)},\right. \\
& \left.\left(\prod_{i=1}^{m}\left(F_{i j}\right)^{\frac{1}{m}}\right) \cdot e^{j 2 \pi\left(\prod_{i=1}^{m}\left(\frac{\phi_{i j}}{2 \pi}\right)^{\frac{1}{m}}\right)}\right\rangle
\end{aligned}
$$

Step 5: Compute the positive distance from average $P D A=\left(P_{i j}\right)_{m \times n}$ and the negative distance from average $N D A=\left(N_{i j}\right)_{m \times n^{\prime}}$, where

$$
\begin{aligned}
P_{i j} & =\frac{\max \left\{0, s\left(a_{i j}\right)-s\left(A V_{j}\right)\right\}}{s\left(A V_{j}\right)} \\
N_{i j} & =\frac{\max \left\{0, s\left(A V_{j}\right)-s\left(a_{i j}\right)\right\}}{s\left(A V_{j}\right)}
\end{aligned}
$$

$s\left(A V_{j}\right)$ and $s\left(a_{i j}\right)$ are score functions of $A V_{j}$ and $a_{i j}$, respectively.

Step 6: Calculate the values of $S P_{i}$ and $S N_{i}$ which denote the weighted sums of PDA and NDA.

$$
\begin{aligned}
& S P_{i}=\sum_{j=1}^{n} w_{j} P_{i j} \\
& S N_{i}=\sum_{j=1}^{n} w_{j} N_{i j}
\end{aligned}
$$

and $w_{j}$ is the weight of the $j-t h$ attribute.

Step 7: Normalize the values $S P_{i}$ and $S N_{i}$ to obtain $N S P_{i}$ and $N S N_{i}$.

$$
\begin{aligned}
& N S P_{i}=\frac{S P_{i}}{\max _{i}\left\{S P_{i}\right\}} \\
& N S N_{i}=1-\frac{S N_{i}}{\max _{i}\left\{S N_{i}\right\}}
\end{aligned}
$$

Step 8: Calculate the appraisal score $A S_{i}(i=1,2, \cdots, m)$ for all alternatives.

$$
A S_{i}=\frac{1}{2}\left(N S P_{i}+N S N_{i}\right)
$$

Step 9: Ranking the alternatives according to the values of $A S_{i}$. The alternative with the highest $A S_{i}$ is the optimal one.

\section{Numerical}

\subsection{The Numerical Example for SVCNS MAGDM Problem}

In this section, we provide a numerical example to select the best green supplier by using the proposed MAGDM approach.

Consider a small-sized trading service and transportation company who wants to seek a langfristig green supplier to purchase a new vehicle for its follow-up operation. The company will assess three potential suppliers $A_{1}, A_{2}, A_{3}$. A managing committee $E$ forms a group of three decision makers $E_{1}, E_{2}, E_{3}$ with different professional skills for the evaluation, and the decision makers' weight vector is $v=\{0.3,0.2,0.5\}$. During the selection process, there are five attributes to consider. They are: 
price/cost $\left(C_{1}\right)$, quality $\left(C_{2}\right)$, delivery $\left(C_{3}\right)$, relationship closeness $\left(C_{4}\right)$ and environmental management systems $\left(C_{5}\right)$; and the corresponding weight vector is $w=\{0.2,0.3,0.25,0.15,0.1\}$. Meanwhile, the five attributes are benefit-type attributes. Three decision makers determined the suitability ratings of three potential suppliers versus the attributes by using the linguistic rating set $S=\{V L, L, F, G, V G\}$ where

$$
\begin{gathered}
V L=\text { VeryLow }=\left(0.2 e^{j(0.8 \pi)}, 0.8 e^{j(1.0 \pi)}, 0.7 e^{j(1.1 \pi)}\right) \\
L=\text { Low }=\left(0.4 e^{j(0.9 \pi)}, 0.6 e^{j(0.9 \pi)}, 0.6 e^{j(1.0 \pi)}\right) \\
F=\text { Fair }=\left(0.6 e^{j(1.0 \pi)}, 0.5 e^{j(0.8 \pi)}, 0.5 e^{j(0.9 \pi)}\right) \\
G=\text { Good }=\left(0.8 e^{j(1.1 \pi)}, 0.4 e^{j(0.7 \pi)}, 0.4 e^{j(0.8 \pi)}\right) \\
V G=\text { VeryGood }=\left(0.9 e^{j(1.2 \pi)}, 0.2 e^{j(0.6 \pi)}, 0.1 e^{j(0.7 \pi)}\right)
\end{gathered}
$$

The three suppliers are to be evaluated with SVCNNs which are listed in Tables 1-3.

Table 1. The evaluated values of decision maker $E_{1}$.

\begin{tabular}{cccccc}
\hline & $C_{1}$ & $C_{2}$ & $C_{3}$ & $C_{4}$ & $C_{5}$ \\
\hline$A_{1}$ & $G$ & $F$ & $L$ & $G$ & $L$ \\
$A_{2}$ & $F$ & $V G$ & $G$ & $F$ & $G$ \\
$A_{3}$ & $V G$ & $V G$ & $F$ & $G$ & $G$ \\
\hline
\end{tabular}

Table 2. The evaluated values of decision maker $E_{2}$.

\begin{tabular}{cccccc}
\hline & $C_{1}$ & $C_{2}$ & $C_{3}$ & $C_{4}$ & $C_{5}$ \\
\hline$A_{1}$ & $F$ & $F$ & $F$ & $F$ & $F$ \\
$A_{2}$ & $F$ & $G$ & $G$ & $F$ & $G$ \\
$A_{3}$ & $G$ & $G$ & $G$ & $V G$ & $F$ \\
\hline
\end{tabular}

Table 3. The evaluated values of decision maker $E_{3}$.

\begin{tabular}{cccccc}
\hline & $C_{1}$ & $C_{2}$ & $C_{3}$ & $C_{4}$ & $C_{5}$ \\
\hline$A_{1}$ & $G$ & $F$ & $L$ & $G$ & $L$ \\
$A_{2}$ & $G$ & $G$ & $G$ & $F$ & $V G$ \\
$A_{3}$ & $V G$ & $G$ & $F$ & $G$ & $G$ \\
\hline
\end{tabular}

To obtain the best green supplier, we utilize the proposed approach to evaluate the three suppliers.

Step 1: Aggregate the information ratings. According to the decision makers' weight vector $v=\{0.3,0.2,0.5\}$, we can obtain the aggregated decision matrix which is shown in Table 4 by using SVCNEWA operator . obtain:

Step 2: Compute the average solution $A V=\left(A V_{j}\right)_{1 \times n}$. According to Equation (11), we can

$$
\begin{aligned}
& A V_{1}=\left(0.8033 e^{j 2 \pi(0.5529)}, 0.3513 e^{j 2 \pi(0.3467)}, 0.2903 e^{j 2 \pi(0.3969)}\right) \\
& A V_{2}=\left(0.7800 e^{j 2 \pi(0.5447)}, 0.3773 e^{j 2 \pi(0.3549)}, 0.3323 e^{j 2 \pi(0.4052)}\right) \\
& A V_{3}=\left(0.6610 e^{j 2 \pi(0.5082)}, 0.4804 e^{j 2 \pi(0.3914)}, 0.4804 e^{j 2 \pi(0.4416)}\right) \\
& A V_{4}=\left(0.7290 e^{j 2 \pi(0.5267)}, 0.4318 e^{j 2 \pi(0.3728)}, 0.4143 e^{j 2 \pi(0.4231)}\right) \\
& A V_{5}=\left(0.7367 e^{j 2 \pi(0.5278)}, 0.4107 e^{j 2 \pi(0.3715)}, 0.3822 e^{j 2 \pi(0.4219)}\right)
\end{aligned}
$$


Table 4. The aggregated decision matrix.

\begin{tabular}{ccc}
\hline Attribute & Alternative & Aggregated Values \\
\hline$C_{1}$ & $A_{1}$ & $\left(0.7689 e^{j 2 \pi(0.5403)}, 0.4187 e^{j 2 \pi(0.3596)}, 0.4187 e^{j 2 \pi(0.4097)}\right)$ \\
& $A_{2}$ & $\left.0.7143 e^{j 2 \pi(0.5255)}, 0.4480 e^{j 2 \pi(0.3744)}, 0.4480 e^{j 2 \pi(0.4245)}\right)$ \\
& $A_{3}$ & $\left(0.8848 e^{j 2 \pi(0.5903)}, 0.2311 e^{j 2 \pi(0.3095)}, 0.1341 e^{j 2 \pi(0.3596)}\right)$ \\
\hline$C_{2}$ & $A_{1}$ & $\left(0.6 e^{j 2 \pi(0.5)}, 0.5 e^{j 2 \pi(0.4)}, 0.5 e^{j 2 \pi(0.45)}\right)$ \\
& $A_{2}$ & $\left(0.8369 e^{j 2 \pi(0.5655)}, 0.3278 e^{j 2 \pi(0.3343)}, 0.2709 e^{j 2 \pi(0.3845)}\right)$ \\
& $A_{3}$ & $\left(0.8369 e^{j 2 \pi(0.5655)}, 0.3278 e^{j 2 \pi(0.3343)}, 0.2709 e^{j 2 \pi(0.3845)}\right)$ \\
\hline$C_{3}$ & $A_{1}$ & $\left(0.4443 e^{j 2 \pi(0.4602)}, 0.5791 e^{j 2 \pi(0.4397)}, 0.5791 e^{j 2 \pi(0.4897)}\right)$ \\
& $A_{2}$ & $\left(0.8 e^{j 2 \pi(0.55)}, 0.4 e^{j 2 \pi(0.35)}, 0.4 e^{j 2 \pi(0.4)}\right)$ \\
$C_{4}$ & $A_{3}$ & $\left(0.6494 e^{j 2 \pi(0.5103)}, 0.4787 e^{j 2 \pi(0.3896)}, 0.4787 e^{j 2 \pi(0.4397)}\right)$ \\
\hline$C_{5}$ & $A_{1}$ & $\left(0.7689 e^{j 2 \pi(0.5403)}, 0.4187 e^{j 2 \pi(0.3596)}, 0.4187 e^{j 2 \pi(0.4097)}\right)$ \\
& $A_{2}$ & $\left(0.5068 e^{j 2 \pi(0.4574)}, 0.5486 e^{j 2 \pi(0.4245)}, 0.5486 e^{j 2 \pi(0.4745)}\right)$ \\
& $A_{3}$ & $\left(0.8253 e^{j 2 \pi(0.5603)}, 0.3506 e^{j 2 \pi(0.3395)}, 0.3095 e^{j 2 \pi(0.3896)}\right)$ \\
\hline$A_{1}$ & $\left(0.4443 e^{j 2 \pi(0.4602)}, 0.5791 e^{j 2 \pi(0.4397)}, 0.5791 e^{j 2 \pi(0.4897)}\right)$ \\
$A_{2}$ & $\left(0.8579 e^{j 2 \pi(0.5755)}, 0.2857 e^{j 2 \pi(0.3242)}, 0.2058 e^{j 2 \pi(0.3744)}\right)$ \\
$A_{3}$ & $\left(0.7689 e^{j 2 \pi(0.5403)}, 0.4187 e^{j 2 \pi(0.3596)}, 0.4187 e^{j 2 \pi(0.4097)}\right)$ \\
\hline
\end{tabular}

Step 3: Calculate the score function of each evaluated value and average solution which is shown in Table 5.

Table 5. The score values of $a_{i j}$ and $A V_{j}$.

\begin{tabular}{cccccc}
\hline & $\boldsymbol{C}_{\mathbf{1}}$ & $\boldsymbol{C}_{\mathbf{2}}$ & $\boldsymbol{C}_{\mathbf{3}}$ & $\boldsymbol{C}_{\mathbf{4}}$ & $\boldsymbol{C}_{\mathbf{5}}$ \\
\hline$A_{1}$ & 0.6171 & 0.5417 & 0.4695 & 0.6171 & 0.4695 \\
$A_{2}$ & 0.5908 & 0.6808 & 0.6333 & 0.4977 & 0.7067 \\
$A_{3}$ & 0.7401 & 0.6808 & 0.5622 & 0.6661 & 0.6171 \\
$A V$ & 0.6618 & 0.6425 & 0.5626 & 0.6023 & 0.6130 \\
\hline
\end{tabular}

Step 4: Computing the matrix of $P D A$ and $N D A$.

$$
P D A=\left[\begin{array}{ccccc}
0 & 0 & 0 & 0.0246 & 0 \\
0 & 0.0596 & 0.1257 & 0 & 0.1529 \\
0.1183 & 0.0596 & 0 & 0.1059 & 0.0067
\end{array}\right]
$$

and

$$
N D A=\left[\begin{array}{ccccc}
0.0675 & 0.1569 & 0.1655 & 0 & 0.2341 \\
0.1073 & 0 & 0 & 0.1737 & 0 \\
0 & 0 & 00007 & 0 & 0
\end{array}\right]
$$

Step 5: Calculate the weighted sums of $P_{i j}$ and $N_{i j}$, denoted as $S P_{i}$ and $S N_{i}$, respectively.

According to the attribute's weight $w=\{0.2,0.3,0.25,0.15,0.1\}$ and Equation (13), we can obtain

$$
\begin{gathered}
S P_{1}=0.0037, S P_{2}=0.0646, S P_{3}=0.0581 \\
S N_{1}=0.1251, S N_{2}=0.0475, S N_{3}=0.0002 .
\end{gathered}
$$


Step 6: Normalize the $S P_{i}$ and $S N_{i}$.

$$
\begin{gathered}
N S P_{1}=0.0573, N S P_{2}=1, N S P_{3}=0.8994 \\
N S N_{1}=0, N S N_{2}=0.6212, N S N_{3}=0.9984 .
\end{gathered}
$$

Step 7: Calculate the appraisal score $A S_{i}$.

$$
A S_{1}=0.0287, A S_{2}=0.8106, A S_{3}=0.9489 .
$$

Obviously, according to the values of $A S_{i}$, we can rank all the alternatives as $A_{3}>A_{2}>A_{1}$ and $A_{3}$ is the best choice.

\subsection{A Comparison Analysis}

In this part, we make a simple comparative analysis. In Section 5.1 we aggregate the information ratings by using SVCNEWA operator, so in this part, we use another operator (SVCNEWG operator) and an EDAS method to obtain the best alternative(s). Meanwhile, we make a comparison through the results obtained by different aggregation operators. According to the steps in Section 4, we can obtain the values of appraisal score and the rankings of alternatives which are shown in Table 6.

Table 6. The values of $A S_{i}$ and the rankings of alternatives.

\begin{tabular}{lcccc}
\hline & $A S_{\mathbf{1}}$ & $A S_{\mathbf{2}}$ & $A S_{3}$ & Ranking \\
\hline SVCNEWA & 0.0287 & 0.8106 & 0.9489 & $A_{3}>A_{2}>A_{1}$ \\
SVCNEWG & 0.0283 & 0.8164 & 0.9386 & $A_{3}>A_{2}>A_{1}$ \\
\hline
\end{tabular}

From the results in Table 6, we can find that the values of appraisal score are slightly different, but the ranking of alternatives and the best alternative are the same, which indicate that the proposed method is practical and effective forthe MAGDM problem.

\section{Conclusions}

In modern enterprises, one of the most significant competitions is for green supply chain management, and green supplier selection is a vital factor of green supply chain management. So how to determine the optimal supplier plays an important role. In real life, green supplier selection can be regarded as a MAGDM problem. Therefore, in this paper, a single-valued complex neutrosophic EDAS model has been established and applied in green supplier selection. Meanwhile, considering that green supplier selection is a group decision making problem, two aggregation operators, namely, a single-valued, complex, neutrosophic, Einstein-weighted average operator and a single-valued, complex, neutrosophic, Einstein-weighted geometric operator have been proposed to fuse overall information ratings into a comprehensive value. Thus, in order to achieve these purposes, this paper firstly introduces the definition of the single-valued complex neutrosophic set and the corresponding operational laws. Next, to fuse overall single-valued complex neutrosophic information, some new aggregation operators of single-valued complex neutrosophic set based on Einstein product and sum, namely, SVCNEWA and SVCNEWA operators, have been proposed. Furthermore, the single-valued complex neutrosophic EDAS model has been established to solve MAGDM problem. Finally, a numerical example for green supplier selection and a comparison have been given. Although the aggregation operators are different in EDAS method, the rankings of alternatives and of the optimal one are the same, which illustrates the practicality and effectiveness of this new model. However, this model only considers the case of known or subjective decision makers' weight vector and attributes' weight vector; it does not discuss the unknown or objective weight vector. So in the future, some models need to be established to obtain a more objective weight vector and more reasonable evaluation 
results. Meanwhile, it is necessary to apply this single-valued complex neutrosophic EDAS method into different fields, such as venture capital, pattern recognition and comprehensive evaluation.

Author Contributions: Methodology, X.C.; validation, H.X.; writing-original draft, X.C.; writing-review and editing, D.X. All authors have read and agreed to the published version of the manuscript.

Funding: This research was funded by the Humanities and Social Sciences Foundation of Ministry of Education of the Peoples Republic of China (17YJA630115).

Conflicts of Interest: The authors declare no conflict of interest.

\section{References}

1. Dai, Z.; Aqlan, F.; Gao, K.; Zhou, Y. A two-phase method for multi-echelon location-routing problems in supply chains. Expert Syst. Appl. 2019, 115, 618-634. [CrossRef]

2. Lo, H.W.; Liou, J.J.; Wang, H.S.; Tsai, Y.S. An integrated model for solving problems in green supplier selection and order allocation. J. Clean. Prod. 2018, 190, 339-352. [CrossRef]

3. Banaeian, N.; Mobli, H.; Fahimnia, B.; Nielsen, I.E.; Omid, M. Green supplier selection using fuzzy group decision making methods: A case study from the agri-food industry. Comput. Oper. Res. 2018, 89, 337-347. [CrossRef]

4. Mumtaz, U.; Ali, Y.; Petrillo, A.; De Felice, F. Identifying the critical factors of green supply chain management: Environmental benefits in Pakistan. Sci. Total Environ. 2018, 640, 144-152. [CrossRef]

5. Zhang, Y.; Tao, F.; Laili, Y.; Hou, B.; Lv, L.; Zhang, L. Green partner selection in virtual enterprise based on Pareto genetic algorithms. Int. J. Adv. Technol. 2013, 67, 2109-2125. [CrossRef]

6. Hosseini, S.; Barker, K. A Bayesian network model for resilience-based supplier selection. Int. J. Prod. Econ. 2016, 180, 68-87. [CrossRef]

7. Wu, Q.; Zhou, L.; Chen, Y.; Chen, H. An integrated approach to green supplier selection based on the interval type-2 fuzzy best-worst and extended VIKOR methods. Inf. Sci. 2019, 502, 394-417. [CrossRef]

8. Liu, P.; Gao, H.; Ma, J. Novel green supplier selection method by combining quality function deployment with partitioned Bonferroni mean operator in interval type-2 fuzzy environment. Inf. Sci. 2019, 490, 292-316. [CrossRef]

9. Qin, J.D.; Liu, X.W.; Pedrycz, W. An extended TODIM multi-criteria group decision making method for green supplier selection in interval type-2 fuzzy environment. Eur. J. Oper. Res. 2017, 258, 626-638. [CrossRef]

10. Gomes, L.; Lima,M. TODIM: Basics and application to multicriteria ranking of projects with environmental impacts. Found. Comput. Decis. Sci. 1992, 16, 113-127.

11. Hwang, C.L.; Yoon, K.P. Multiple Attribute Decision Making; Springer: Berlin, Germany, 1981.

12. Opricovic S. Multicriteria Optimization of Civil Engineering Systems; Faculty of Civil Engineering: Belgrade, Serbia, 1998; Volume 2, pp. 5-21.

13. Govindan, K.; Jepsen, M.B. ELECTRE: A comprehensive literature review on methodologies and applications. Eur. J. Oper. Res. 2016, 250, 1-29. [CrossRef]

14. Pamucar, D.; Cirovic, G. The selection of transport and handling resources in logistics centers using multi-attributive border approximation area comparison (mabac). Expert Syst. Appl. 2015, 42, 3016-3028. [CrossRef]

15. Ji, P.; Wang, J.Q.; Zhang, H.Y. Selecting an outsourcing provider based on the combined MABAC-ELECTRE method using single-valued neutrosophic linguistic sets. Comput. Ind. Eng. 2018, 120, 429-441. [CrossRef]

16. Keshavarz Ghorabaee, M.; Zavadskas, E.K.; Olfat, L.; Turskis, Z. Multi-criteria inventory classification using a new method of evaluation based on distance from average solution (EDAS). Informatica 2015, 26, 435-451. [CrossRef]

17. Keshavarz Ghorabaee K.; Zavadskas E.K.; Amiri M.; Turskis Z. Extended EDAS method for fuzzy multi-criteria decision-making: An application to supplier selection. Int. J. Comput. Commun. Control 2016, 3, 358-371. [CrossRef]

18. Kahraman C.; Ghorabaee M.K.; Zavadskas E.K. Intuitionistic fuzzy EDAS method: An application to solid waste disposal site selection. J. Environ. Eng. Landsc. Manag. 2017, 25, 1-12. [CrossRef]

19. Karaşan, A.; Kahraman, C. A novel interval-valued neutrosophic EDAS method: Prioritization of the United Nations national sustainable development goals. Soft Comput. 2018, 22, 4981-4906. [CrossRef] 
20. Zadeh, L.A. Fuzzy sets. Inf. Control 1965, 8, 338-353. [CrossRef]

21. Atanassov, K.T. Intuitionistic fuzzy sets. Fuzzy Sets Syst. 1986, 20, 87-96. [CrossRef]

22. Smarandache, F. A unifying field in logics: Neutrosophic logic. Neutrosophy, neutrosophic set, neutrosophic probability and statistics. Multiple-Valued Logic 2001, 95, 5-21.

23. Wang, H.; Smarandache, F.; Zhang, Y.; Sunderraman, R. Single valued neutrosophic sets. Rev. Air Force Acad. 2010, 10, 410-413.

24. Sunderraman, R.; Wang, H. Interval neutrosophic sets and logic: Theory and applications in computing. arXiv 2005, arXiv:cs/0505014v1.

25. Ye, J. A multicriteria decision-making method using aggregation operators for simplified neutrosophic sets. J. Intell. Fuzzy Syst. 2014, 26, 2459-2466. [CrossRef]

26. Wang, J.Q.; Li, X.E. TODIM method with multi-valued neutrosophic sets. Control Decis. 2015, 30, 1139-1142.

27. Ali, M.; Smarandache, F. Complex neutrosophic set. Neural Comput. Appl. 2017, 28, 1817-1834. [CrossRef]

28. Al-Quran, A.; Alkhazaleh, S. Relations between the complex neutrosophic sets with their applications in decision making. Axioms 2018, 7, 64. [CrossRef]

29. Quek, S.G.; Broumi, S.; Selvachandran, G.; Bakali, A.; Talea, M.; Smarandache, F. Some results on the graph theory for complex neutrosophic sets. Symmetry 2018, 10, 190. [CrossRef]

30. Sudharsan, S.; Ezhilmaran, D. Weighted arithmetic average operator based on interval-valued intuitionistic fuzzy values and their application to multi criteria decision making for investment. J. Inf. Optim. Sci. 2016, 37, 347-260. [CrossRef]

31. Wang, J.Q.; Han, Z.Q.; Zhang, H.Y. Multi-criteria group decision-making method based on intuitionistic interval fuzzy information. Group Decis. Negot. 2014, 23, 715-734. [CrossRef]

32. Yager, R.R. On ordered weighted averaging aggregation operators in multi -criteria decision making. IEEE Trans. Syst. Man Cybern. 1988, 18, 183-190. [CrossRef]

33. Kacprzyk, J.; Zadrożny, S. Linguistic summarization of the contents of Web server logs via the Ordered Weighted Averaging (OWA) operators. Fuzzy Sets Syst. 2016, 285, 182-198. [CrossRef]

34. Zhou, L.G.; Chen, H.Y. Continuous generalized OWA operator and its application to decision making. Fuzzy Sets Syst. 2011, 168, 18-34. [CrossRef]

35. Liu, P.; Teng, F. Multiple criteria decision making method based on normal interval-valued intuitionistic fuzzy generalized aggregation operator. Complexity 2016, 21, 277-290. [CrossRef]

36. Ji, P.; Wang, J.Q.; Zhang, H.Y. Frank prioritized Bonferroni mean operator with single-valued neutrosophic sets and its application in selecting third-party logistics providers. Neural Comput. Appl. 2018, 30, 799-823. [CrossRef]

37. Yu, D. Intuitionistic fuzzy geometric Heronian mean aggregation operators. Appl. Soft Comput. 2013, 13, 1235-1246. [CrossRef]

38. Klement, E.P.; Mesiar, R.; Pap, E. Triangular norms. Position paper I: Basic analytical and algebraic properties. Fuzzy Sets Syst. 2004, 143, 5-26. [CrossRef]

39. Li, B.; Wang, J.; Yang, L.; Li, X. A Novel Generalized Simplified Neutrosophic Number Einstein Aggregation Operator. IAENG Int. J. Appl. Math. 2018, 48, 67-72.

40. Peng, J.J.; Wang, J.Q.; Wu, X.H.; Wang, J.; Chen, X.H. Multi-valued neutrosophic sets and power aggregation operators with their applications in multi-criteria group decision-making problems. Int. J. Comput. Intell. Syst. 2015, 8, 345-363. [CrossRef]

41. Zhao, X.; Wei, G. Some intuitionistic fuzzy Einstein hybrid aggregation operators and their application to multiple attribute decision making. Knowl.-Based Syst. 2013, 37, 472-479. [CrossRef]

(c) 2020 by the authors. Licensee MDPI, Basel, Switzerland. This article is an open access article distributed under the terms and conditions of the Creative Commons Attribution (CC BY) license (http://creativecommons.org/licenses/by/4.0/). 